\title{
Review Article NNK-Induced Lung Tumors: A Review of Animal Model
}

\author{
Hua-Chuan Zheng and Yasuo Takano \\ Kanagawa Cancer Center, Clinical Research Institute, Yokohama 241-0815, Japan \\ Correspondence should be addressed to Yasuo Takano, ytakano@gancen.asahi.yokohama.jp
}

Received 10 September 2010; Revised 27 December 2010; Accepted 20 January 2011

Academic Editor: Aditi Chatterjee

Copyright ( 92011 H.-C. Zheng and Y. Takano. This is an open access article distributed under the Creative Commons Attribution License, which permits unrestricted use, distribution, and reproduction in any medium, provided the original work is properly cited.

\begin{abstract}
The incidence of lung adenocarcinoma has been remarkably increasing in recent years due to the introduction of filter cigarettes and secondary-hand smoking because the people are more exposed to higher amounts of nitrogen oxides, especially 4-(methylnitrosamino)-1-(3-pyridyl)-1-butanone(NNK), which is widely applied in animal model of lung tumors. In NNKinduced lung tumors, genetic mutation, chromosome instability, gene methylation, and activation of oncogenes have been found so as to disrupt the expression profiles of some proteins or enzymes in various cellular signal pathways. Transgenic animal with specific alteration of lung cancer-related molecules have also been introduced to clarify the molecular mechanisms of NNK in the pathogenesis and development of lung tumors. Based on these animal models, many antioxidant ingredients and antitumor chemotherapeutic agents have been proved to suppress the NNK-induced lung carcinogenesis. In the future, it is necessary to delineate the most potent biomarkers of NNK-induced lung tumorigenesis, and to develop efficient methods to fight against NNK-associated lung cancer using animal models.
\end{abstract}

\section{Introduction}

Throughout the spectrum of cancers worldwide, lung cancer claims the lives of over one million people worldwide each year and is one of the most common and lethal cancers of men and women in North America, Europe, and East Asia although current strategies in the treatment of lung cancer including surgery, radiation therapy, chemotherapy, and targeted biological therapies have slightly generated an increase in the 5-year survival rate for all stages combined [1]. The secular trend in lung cancer histology indicates that the proportion or incidence of lung adenocarcinoma has been increasing markedly over the past two decades, surpassing the squamous cell carcinoma as the most common histological subtype of lung cancer in many countries, which is partly due to the introduction of filter cigarettes and secondary-smoking because the people are more exposed to higher amounts of nitrogen oxides, nitrosated compounds, and lung-specific smoke carcinogens [1-3].

Epidemiological and laboratory evidences demonstrate a strong etiological association with smoking, which contains volatile $\mathrm{N}$-nitrosamines such as $\mathrm{N}$-nitrosodimethylamine, $\mathrm{N}$-nitrosopyrrolidine as well as tobacco-specific
$\mathrm{N}$-nitrosamines such as $\mathrm{N}^{\prime}$-nitrosonornicotine and 4(methylnitrosamino)-1-(3-pyridyl)-1-butanone (NNK). Although $\mathrm{N}^{\prime}$-nitrosonornicotine causes tumors of the oesophagus and nasal cavity in rats, NNK reproducibly induces pulmonary adenocarcinomas (PAC) in laboratory rodents, including rats, mice, hamsters, and ferrets, which therefore has been classified as a human lung carcinogen by the International Agency for Research on Cancer working group. Significant incidences of tumors occurred in the lungs of strain $\mathrm{A} / \mathrm{J}$ progeny $(24 \mathrm{wk}$ ) and in the livers of male C3B6F1 and Swiss progeny (72 wk) after NNK treatment [4]. NNK is known to be activated in the lung via $\alpha$-carbon hydroxylation by cytochrome P450 (CYP), hemoglobin, and lipoxygenases (LOX) [5]. The production of methylating and pyridyloxobutylating agents that attack DNA and cause the genetic changes is known to be associated with self sufficiency in growth signals, evasion of apoptosis, insensitivity to antigrowth signals, sustained angiogenesis, tissue invasion and metastasis, and limitless replicative potential.

With lung carcinogenesis models, it may be helpful to gain insights into basic biology of lung tumors, find out markers for early diagnosis, and validate antilung cancer 
prevention and therapies. Ferrets exposed to both NNK and smoke developed preneoplastic lesions (squamous metaplasia, dysplasia, and atypical adenomatous hyperplasia) with complex growth patterns and further exposure will cause squamous cell carcinoma, adenosquamous carcinoma, and adenocarcinoma $[6,7]$. In A/J mice, 14 -week NNK treatment can cause pulmonary hyperplasias along the alveolar septa, in which the proliferating cells showed cuboidal shape, lamellar bodies, and centrally localized ovoid nuclei as type II pneumocytes. From 34 to 42 wks after treatment, progression to neoplasia was characterized by a declined hyperplasias and an increased adenoma. Carcinomas appeared to increase in frequency 34 wks after NNK treatment and comprised more than $50 \%$ of the pulmonary lesions by 54 wks. The growth pattern of carcinomas began to change from solid to mixed (solid and papillary) 42 wks after NNK treatment [8].

\section{Mechanisms of NNK-Induced Lung Carcinogenesis}

The establishment of genetic and epigenetic alteration followed by gene expression profiling is of great use and help to clarify molecular mechanisms of NNK-induced lung tumorigenesis. In experimental model, NNK could cause Adrb2 SNP mutation of Syrian golden hamsters, and Kras mutation in codon 12 of the A/J mice [9-11]. Bacterial artificial chromosome array-based comparative genomic hybridization indicated that the gains on chromosomes 6 and 8 , and losses on chromosomes 11 and 14, were more common in NNK-induced tumors and the changes on chromosomes $8,11,12$, and 14 were positively associated with the degree of chromosome instability $[12,13]$. The methylation of the retinoic acid receptor (RAR) $\beta$ and death-associated protein kinase gene was also detected in preneoplastic hyperplasias or adenocarcinoma induced by NNK treatment [14-16].

NNK administration reduced the miR-126* expression targeting CYP2A3 in rat lungs, but induced CYP2A3 expression [17]. The 14-3-3 isoforms $(\theta, \xi$, and $\sigma)$ and annexin A5 were significantly downregulated in NNK-induced pulmonary adenocarcinogenesis of $\mathrm{A} / \mathrm{J}$ mice according to $2 \mathrm{D}$ electrophoresis [18]. Immunohistochemically, NNK induced preneoplastic lesions in lungs, including alveolar hyperplasia and atypical dysplasia with COX-2 and PCNA overexpression [19]. In lung adenocarcinoma of hamsters, the overexpression of PKA, cAMP, CREB and phosphorylated CREB in $\beta 2$-adrenergic receptor pathway and EGFR-specific phosphorylated tyrosine kinase, Raf-1 and ERK1/2 and their phosphorylated forms in EGFR pathway were observed [20]. Regarding the control of cell cycle, there was an upregulated expression of cyclin D1 and cdk4, but downregulated Rb expression in NNK-induced lung adenomas and adenocarcinomas [21]. Exposure of NNK to hamsters and mice led to the decreased expression of Clara cell $10-\mathrm{kDa}$ protein (CC10) which subsequently enhances the induction of anchorageindependent growth in response to NNK [22]. The NNK treatment enhanced the expression of fatty acid synthase, transketolase, pulmonary surfactant-associated protein $\mathrm{C}$,
L-plastin, annexin A1, and haptoglobin, but the expression of transferrin, $\alpha$-1-antitrypsin, and apolipoprotein A1 decreased [23]. The NNK-mediated expression of protein and RNA in mouse lung tumors will provide more information and clues about markers of NNK-induced tumroigenesis and targets for antipreventive agents in NNK-related lung cancer.

\section{Transgenic Animal Model of NNK-Mediated Lung Carcinoma}

Transgenic models have the potential to play an important role in identification of potential human carcinogens and clarify the molecular mechanisms of carcinogens in the pathogenesis and development of malignancies. Actually, many transgenic and knockout mice have been applied to investigate the NNK-induced lung carcinogenesis.

When transgenic mice that overexpress HGF in the airway epithelium were exposed to NNK, they exhibited congestion in the alveolar spaces, excessive production of blood vessels, a convoluted pattern of airways with more number of lung tumors, and high tumor incidence, compared with control [24]. All NNK-treated SPC/myc transgenic mice showed bronchioloalveolar hyperplasia and adenocarcinoma formation [25]. The p53 mutation, on an A/J F1 background, were more susceptible to NNK and mice with a mutant p53 developed larger lung tumors, emphasizing the potential effects of a p53 mutation both on tumor initiation and progression [26]. NNK-treated mice expressing high levels of IGF-IR transgene developed larger tumors than the control mice [27].

Galectin-3, a $\beta$-galactoside-binding lectin is a multifunctional protein, which regulates cellular adhesion, proliferation, and apoptosis, and in turn contributes to tumorigenesis. We intraperitoneally administrated NNK into galectin3 wild-type $($ gal3+/+) and knockout (gal3-/-) mice and found that the incidence of lung tumors was significantly lower in gal3 $-/-$ than gal3 $+/+$ mice after 32 wks. Compared with gal $3+/+$ mice, pathway analysis of gene microarray indicated that galectin-3 upregulated carcinogenesisrelated genes (e.g., B-cell receptor, ERK/MAPK, and PPAR signalings) in normal condition, and NNK-induced gene expression were associated with cellular growth (e.g., Wnt/ $\beta$ catenin signaling) or immunological disease (e.g., EGF and PDGF signalings) in lung carcinogenesis regardless of galectin-3 status. The functions involved in NNK-induced PAC include cellular growth and proliferation and canonical pathways for Wnt/ $\beta$-catenin signaling $[28,29]$.

Human methylguanine-DNA methyltransferase (MGMT) transgenic mice overexpressing $\mathrm{O}^{6}$-alkylguanineDNA alkyltransferase (AGT) in lung were crossbred with A/J mice for animal model of lung tumors. The MGMT transgenic mice had higher AGT activity, lower multiplicity, and smaller-sized lung tumors than the control mice after NNK treatment. Moreover, a reduction in K-ras mutations in lung tumors was found in the MGMT transgenic mice [30]. When MGMT-/- mice were crossed with a lacI-based transgenic reporter line, NNK-dependent lacI mutations 
was more frequently observed in MGMT-/- tissues. The mutational spectra of NNK-treated MGMT-/- lungs revealed an increase in $\mathrm{G}: \mathrm{C}$ to $\mathrm{A}: \mathrm{T}$ changes accompanied by a shift from $\mathrm{CpG}$ to GpG sites [31]. 8-hydroxyguanine DNA glycosylase 1 (Ogg1) gene encodes an enzyme that repairs an oxidative DNA injury 8-oxoguanine (8-oxoG), whose deficiency results in the development of lung adenomas and preneoplastic atypical hypreplasias in knockout mice treated with NNK [32].

There was a NNK-dose-dependent increase in lung tumor size in PTEN $+/-$, compared with $+/+$ mice. Lung tumors from PTEN+/- mice had K-ras mutations, low PTEN expression, and Akt pathway activation [33]. Although mice with a knockout of G-protein coupled receptor 5A develop lung tumors after a long latent period, NNK treatment could hurry the development of lung tumors, exhibiting increased tumor incidence and multiplicity and a dramatic increase in lesion size [34]. The 4 eukaryotic initiation factor (ebp)1-/-/4ebp2-/- mice showed increased sensitivity to NNK-induced tumorigenesis, compared with the wild-type counterparts due to translational activation of genes governing angiogenesis, growth and proliferation and translational activation of CYP2A5 [35]. NNK exposure of CC1-knockout mice causes a significantly higher incidence of airway epithelial hyperplasia and lung adenomas with $\mathrm{K}$-ras mutation, Fas ligand expression and MAPK/ERK phosphorylation increased than wild-type littermates [36].

The numbers of NNK-induced lung tumors and tumor multiplicity were reduced in the lung-NADPH-P450reductase (Cpr)-null mice, relative to wild-type mice, which was correlated with lower lung O6-methylguanine adduct levels. Lung tumors in lung-Cpr-null mice were positive for CPR expression, indicating that the tumors did not originate from Cpr-null cells [37]. With the NNK treatment, the tumor multiplicity in angiotensin II type 2 receptor (AT2)-null mice was significantly smaller than that in wild-type mice [38].

\section{Repressing of NNK-Induced Lung Carcinogenesis}

The antioxidants (Selenium, $\beta$-carotene, $\mathrm{N}$-acetylcysteine, and $\alpha$-tocopherol) from tea, plant, or vitamin can prevent lung carcinogenesis in an NNK-treated ferret model by preventing oxidative DNA damage, and increasing the levels of lung retinoic acid in the lung cancer induced by NNK. Chemotherapeutic agents also play a preventive role in the NNK-induced lung tumorigenesis due to the modification of disrupted signal pathways.

4.1. Selenium. 1,4-phenylenebis(methylene)selenocyanate (p-XSC) was highly effective to inhibit the initiation and postinitiation phase of lung tumorigenesis induced by NNK in $\mathrm{A} / \mathrm{J}$ mice and reduce NNK-induced DNA methylation and 8-hydroxy- $2^{\prime}$-deoxyguanosine $(8-\mathrm{OHdG})$ levels in the lung [39]. The levels of protein-bound:free GSH ratios and Cys ratios were significantly decreased, and total glutathione S-transferase (GST) enzyme activity, as well as GST-pi and GST-mu enzyme activities, glutathione peroxidase (GPX) activity were significantly induced in p-XSC-treated mice after NNK treatment. These results suggest that $\mathrm{p}$-XSC inhibits tumor formation partially by protecting against oxidative damage [40]. Additionally, p-XSC was shown to significantly inhibit formation of O6-methylguanine and 7 -methylguanine in the mouse lungs treated with NNK, indicating its inhibitory role in DNA methylation [41]. Additionally, 2-oxo-selenazolidine-4(R)-carboxylic acids and selenocystine significantly reduced lung adenoma multiplicity in NNK-treated mice with hepatic selenium levels elevated [42].

4.2. Tea. The inhibitory effect of tea on lung carcinogenesis has been attributed to its major ingredients, such as polyphenolic compound, epigallocatechin gallate (EGCG), caffeine, thearubigins, and theaflavins because tea inhibited the formation of reactive oxygen species and radicals, and induced CYP1A1, $1 \mathrm{~A} 2$ and 2B1, and glucuronosyl transferase [43]. In NNK-induced lung tumors, tea treatment inhibited angiogenesis, as indicated by the lower microvessel density and enhanced the apoptosis index labeled by TUNEL [44]. The levels of 8-hydroxydeoxyguanosine, a marker of oxidative DNA damage, were significantly suppressed in NNK-induced mice treated with green tea or EGCG. The oxidation products found in black tea, thearubigins, and theaflavins, also possessed antioxidant activity and retarded the development of lung cancer caused by NNK [45]. The administration of Polyphenon E and Caffeine not only reduced the incidence and multiplicity of lung adenocarcinoma in female $\mathrm{A} / \mathrm{J}$ mice induced by NNK, but also inhibited cell proliferation, enhanced apoptosis, and lowered levels of c-Jun and ERK1/2 phosphorylation in adenocarcinomas and adenomas, suggesting that tea polyphenols (and perhaps caffeine) inhibit NNK-induced lung tumorigenesis [46].

4.3. Vitamin. Vitamin $\mathrm{E}$ inhibits tumor cell growth in vitro irrespective of its antioxidative effect. In NNK-induced lung tumors of mice, Vitamin E supplement reduced the mutation frequency of K-ras at codon 12, suggesting that it suppresses NNK-induced DNA injury [47]. $\alpha$-tocopheryloxybutyric acid (TSE), a nonantioxidative vitamin E derivative, could inhibit cell proliferation during the mouse lung tumorigenic process treated with NNK. The administration of Vitamin E or TSE suppressed the labeling index of the PCNA, the elevation of ornithine decarboxylase activity at a promotion phase of NNK-induced lung tumorigenesis $[48,49] . \gamma$ tocopherol-rich mixture of tocopherols $(\gamma$-TmT, considered as vitamin E) significantly lowered tumor multiplicity, tumor volume, and tumor burden, which was associated with high apoptosis and low levels of 8-hydroxydeoxyguanine, $\gamma$-H2AX and nitrotyrosine in the NNK-induced lung [50].

Mice receiving the supplementation of $1 \alpha, 25$-dihydroxyvitamin D3 $(1,25 \mathrm{D})$ had significantly lower tumor incidence and tumor multiplicity, but experienced body weight loss, kidney calcium deposition, elevated kidney CYP24 expression, and decreased fasting plasma 1,25D levels [51]. Inhaled mid-dose isotretinoin (13-cis-retinoic acid) caused up-regulation of lung tissue nuclear RARs relative 
to vehicle-exposed mice [52]. 9-cis-Retinoic acid (9cRA) binds both RARs and retinoid $\mathrm{X}$ receptors and has been shown to be a potential chemopreventive agent. The mice receiving 9cRA supplementation had significantly lower tumor multiplicity and showed a trend toward lower tumor incidence, as compared with the mice given NNK alone [53]. The mice exposed to the high isotretinoin (13-cis retinoic acid) dose or 5-hydroxy-4-(2-phenyl-(E) ethenyl)$2(5 \mathrm{H})$-furanone (KYN-54, a novel retinoidal butenolide compound) showed reductions of tumor multiplicity after NNK treatment [54].

4.4. Plant and Vegetable. The discovery of dietary-related compounds with potential to inhibit lung cancer may present promising and practical approaches to reduce the risk of lung cancer caused by smoking. The exposure to fermented brown rice and rice bran significantly reduced the multiplicity, and tumor size of NNK-induced lung tumor with the expression of CYP 2A5 mRNA and Ki-67 protein decreased [55]. Deguelin, a natural plant product, specifically inhibits the proliferation of premalignant and malignant bronchial epithelial cells by blocking Akt activation [56]. Feeding with powdered adlay seed exerts an anticancer effect, evidenced by the reduced number of surface lung tumors [57]. The administration of Changkil saponins suppressed the NNK-induced increase in the level of PCNA and the number of lung tumors [58]. The treatment of 7-hydroxy-3methoxycadalene from Zelkova serrata significantly reduced the incidence of adenomas and adenocarcinoma in a concentration-dependent manner [59]. Cinnamaldehyde (CNMA) treatment significantly reduced the combined incidence of adenomas and carcinomas, tumor multiplicity in transgenic rasH2 male mice [10]. Kava is a traditional beverage in the South Pacific islands and could prevent NNK plus BaP-induced lung tumorigenesis in A/J mice by enhancing apoptosis, inhibiting proliferation and the activation of NF-kappaB in lung tumors [60]. Isothiocyanates are derived from cruciferous vegetables and their $\mathrm{N}$-acetylcysteine and phenethyl conjugates inhibit the formation of lung adenoma and adenocarcinoma with a significant reduction in PCNA and an induction of apoptosis in $\mathrm{A} / \mathrm{J}$ mice induced by NNK $[61,62]$. In (A/J × TSG-p53 "knockout") F1 mice with either the p53+/- or p53+/+ genotype, phenethyl isothiocyanate (PEITC) pretreatment significantly decreased tumor incidence and multiplicity [63]. $\beta$-carotene increased lung tumor multiplicity, lung tumor size, blood cell cAMP, serum, and lung levels of retinoids and induced $\mathrm{p}-\mathrm{CREB}$ and p-ERK1/2 in PAC induced by NNK [64]. Treatments with Satsuma mandarin juice (MJ), MJ2, and MJ5 reduced the incidence and multiplicity of NNK-induced lung tumors by decreasing PCNA-positive index in lung tumors [65].

4.5. Enzyme Inhibitor. CYP enzymes can catalyze the $\alpha$ hydroxylation of NNK for its activation in the oxidative metabolism pathway, such as CYP2A6. A trend was noted for 8-Methoxypsoralen (8-MOP), an inhibitor of CYP2A6, to reduce adenomas and adenocarcioma to a greater extent than hyperplasia in mouse lung treated by NNK [66-69]. NNK is reported to promote COX-2 activity in colon and gastric cancer cells and the development of NNK-induced adenocarcinomas in mice is reduced by inhibitors of cyclooxygenase [70]. Another report showed that such specific COX-2 inhibitors as acetylsalicylic acid or N-[2-(cyclohexyloxy)4-nitrophenyl]-methanesulfona-mide significantly increased the apoptotic index and inhibited the expression of COX2 in NNK-treated mice [71]. Farnesyltransferase inhibitors (FTIs) included manumycin, gliotoxin, dihydroepiandrosterone, perillyl alcohol, and FTI-276. FTI-276 reduced both the tumor multiplicity and the total tumor volume/burden per mouse. The apoptotic index in FTI-276-treated tumors showed an increase of $77 \%$ over control tumors [72].

4.6. Fatty Acid. The supplementation of fish oil with a low $\omega-6(n-6) / \omega-3(n-3)$ polyunsaturated fatty acid ratio was able to significantly decrease lung tumor prevalence compared to groups receiving soybean oil and corn oil, which was associated with increased expression of cell cycle inhibitor p21Cip1 and lipoxygenase isoform 15-LOX in the lungs [73]. The treatment of NNK increased the level of prostaglandin E2 as well as PCNA and induced the activation of an ERK cascade (ERK, MEK, and Raf-1) in high linoleic acid oil- (LA-) fed mice. On the other hand, oleic acid oil (OA) feeding abolished the NNK-induced activation of the ERK cascade. In conjugation with these events, OA feeding reduced lung tumor incidence and tumor multiplicity in mice compared with LA feeding. These results suggest that OA suppresses lung tumorigenesis and that this suppression is correlated with the inhibition of PGE2 production and inactivation of the ERK cascade [74]. Myoinositol in AIN-93 diet also proved to reduce the development of lung tumors induced by NNK [75].

4.7. Anticancer Reagents. The anticancer chemicals targeting the cell signals and metabolism can be employed to prevent carcinogenesis. Gefitinib is an EGFR tyrosine kinase inhibitor (EGFR-TKI) and could significantly suppress the multiplicities of the NNK-induced tumors in a dosedependent manner [76]. The administration of Rapamycin, an inhibitor of mTOR, decreased tumor size, proliferative rate, tumor multiplicity, and mTOR activity in NNK-treated mice [77]. Targretin is a retinoid specifically selective for retinoid $\mathrm{X}$ receptors and widely used as an anticancer reagent. In mice, it could decrease the multiplicity and size of NNK-induced tumors, demonstrating its preventive and therapeutic activity [78]. Histone deacetylase inhibitors, such as suberoylanilide hydroxamic acid (SAHA), showed a significant inhibition of lung tumor multiplicity in mice treated with NNK. However, a significant inhibition of the alpha-hydroxylation pathway of NNK was observed in lung microsomes, suggesting that SAHA may act to inhibit the activation pathways of NNK metabolism [79].

\section{Future Perspectives}

The contribution of NNK to the imbalance between cellular proliferation and apoptosis, and subsequent lung tumorigenesis has been consistently described and confirmed in 
numerous animal models. According to animal experiments, several mechanisms of NNK-induced lung carcinoma have been proposed, including (i) the activation of oncogenes via mutation, (ii) interruption and/or silencing of genes encoding enzymes coupled with NNK, (iii) direct manipulation of enzymes (specifically from the CYP protein family) responsible for activation and initiation of NNK-mediated processes, and (iv) the disruption of the signal pathways. Some primary preventive approaches have not yet been established, including (i) rendering NNK inactivation by antioxidants (tea, vegetable, vitamin and metal compunds) and (ii) obstructing the function of NNK (anticancer chemotherapeutic agents). In the recent years, too many transgenic mice has been bred and applied in the establishment of lung cancer model, which can provide an efficient tool for the investigation of lung cancer without the influence of chemical carcinogen. If treated with NNK, it is of great help and use to clarify the molecular mechanism of NNK-induced lung carcinogenesis and find out the novel target to prevent NNK-associated lung cancer. Therefore, it is necessary to delineate the most potent biomarkers of NNK-induced lung tumorigenesis, and to develop efficient methods to fight against this kind of lung cancer using animal model in the future.

\section{Acknowledgments}

This study was supported by Grant-in-Aid for Scientific Research from the Ministry of Education, Culture, Sports and Technology of Japan (20659109), Japanese Smoking Research Foundation, Japanese Uehara Memorial Foundation, and Research Grants for JSPS Postdoctoral Fellows.

\section{References}

[1] A. J. Alberg, M. V. Brock, and J. M. Samet, "Epidemiology of lung cancer: looking to the future," Journal of Clinical Oncology, vol. 23, no. 14, pp. 3175-3185, 2005.

[2] H. Zheng, H. O. Abdel Aziz, Y. Nakanishi et al., "Oncogenic role of JC virus in lung cancer," Journal of Pathology, vol. 212, no. 3, pp. 306-315, 2007.

[3] D. Hoffmann, A. Rivenson, and S. S. Hecht, "The biological significance of tobacco-specific N-nitrosamines: smoking and adenocarcinoma of the lung," Critical Reviews in Toxicology, vol. 26, no. 2, pp. 199-211, 1996.

[4] L. N. Anderson, S. S. Hecht, D. E. Dixon et al., "Evaluation of the transplacental tumorigenicity of the tobacco-specific carcinogen 4-(methylnitrosamino)-1-(3-pyridyl)-1-butanone in mice," Cancer Research, vol. 49, no. 14, pp. 3770-3775, 1989.

[5] L. L. Bedard, G. B. J. Smith, K. R. Reid, D. Petsikas, and T. E. Massey, "Investigation of the role of lipoxygenase in bioactivation of 4-(methylnitrosamino)-1-(3-pyridyl)-1butanone (NNK) in human lung," Chemical Research in Toxicology, vol. 15, no. 10, pp. 1267-1273, 2002.

[6] Y. Kim, F. Lian, K. J. Yeum et al., "The effects of combined antioxidant ( $\beta$-carotene, $\alpha$-tocopherol and ascorbic acid) supplementation on antioxidant capacity, DNA single-strand breaks and levels of insulin-like growth factor-1/IGF-binding protein 3 in the ferret model of lung cancer," International Journal of Cancer, vol. 120, no. 9, pp. 1847-1854, 2007.
[7] Y. Kim, N. Chongviriyaphan, C. Liu, R. M. Russell, and X.D. Wang, "Combined antioxidant $(\beta$-carotene, $\alpha$-tocopherol and ascorbic acid) supplementation increases the levels of lung retinoic acid and inhibits the activation of mitogen-activated protein kinase in the ferret lung cancer model," Carcinogenesis, vol. 27, no. 7, pp. 1410-1419, 2006.

[8] S. A. Belinsky, T. R. Devereux, J. F. Foley, R. R. Maronpot, and M. W. Anderson, "Role of the alveolar type II cell in the development and progression of pulmonary tumors induced by 4-(methylnitrosamino)-1-(3-pyridyl)-1-butanone in the A/J mouse," Cancer Research, vol. 52, no. 11, pp. 3164-3173, 1992.

[9] T. Masi, M. Cekanova, K. Walker et al., "Nitrosamine 4(methylnitrosamino)-1-(3-pyridyl)-1-butanone-induced pulmonary adenocarcinomas in Syrian golden hamsters contain beta 2-adrenergic receptor single-nucleotide polymorphisms," Genes Chromosomes and Cancer, vol. 44, no. 2, pp. 212-217, 2005.

[10] T. Imai, K. Yasuhara, T. Tamura et al., "Inhibitory effects of cinnamaldehyde on 4-(methylnitrosamino)-1-(3-pyridyl)-1butanone-induced lung carcinogenesis in rasH2 mice," Cancer Letters, vol. 175, no. 1, pp. 9-16, 2002.

[11] A. Hisamoto, E. Kondo, K. Kiura et al., "Point mutation of Kras gene in cisplatin-induced lung tumours in A/J mice," Lung Cancer, vol. 58, no. 1, pp. 15-20, 2007.

[12] C. R. Herzog, D. Desai, and S. Amin, "Array CGH analysis reveals chromosomal aberrations in mouse lung adenocarcinomas induced by the human lung carcinogen 4(methylnitrosamino)-1-(3- pyridyl)-1-butanone," Biochemical and Biophysical Research Communications, vol. 341, no. 3, pp. 856-863, 2006.

[13] C. R. Herzog, N. Bodon, B. Pittman et al., "Carcinogenspecific targeting of chromosome 12 for loss of heterozygosity in mouse lung adenocarcinomas: implications for chromosome instability and tumor progression," Oncogene, vol. 23, no. 17, pp. 3033-3039, 2004.

[14] B. R. Vuillemenot, L. C. Pulling, W. A. Palmisano, J. A. Hutt, and S. A. Belinsky, "Carcinogen exposure differentially modulates RAR- $\beta$ promoter hypermethylation, an early and frequent event in mouse lung carcinogenesis," Carcinogenesis, vol. 25, no. 4, pp. 623-629, 2004.

[15] L. C. Pulling, B. R. Vuillemenot, J. A. Hutt, T. R. Devereux, and S. A. Belinsky, "Aberrant promoter hypermethylation of the death-associated protein kinase gene is early and frequent in murine lung tumors induced by cigarette smoke and tobacco carcinogens," Cancer Research, vol. 64, no. 11, pp. 3844-3848, 2004.

[16] B. R. Vuillemenot, L. C. Pulling, W. A. Palmisano, J. A. Hutt, and S. A. Belinsky, "Carcinogen exposure differentially modulates RAR- $\beta$ promoter hypermethylation, an early and frequent event in mouse lung carcinogenesis," Carcinogenesis, vol. 25, no. 4, pp. 623-629, 2004.

[17] S. Kalscheuer, X. Zhang, Y. Zeng, and P. Upadhyaya, "Differential expression of microRNAs in early-stage neoplastic transformation in the lungs of F344 rats chronically treated with the tobacco carcinogen 4-(methylnitrosamino)-1-(3pyridyl)-1-butanone," Carcinogenesis, vol. 29, no. 12, pp. 2394-2399, 2008.

[18] J. D. Bortner Jr., A. Das, T. M. Umstead et al., "Downregulation of 14-3-3 isoforms and annexin A5 proteins in lung adenocarcinoma induced by the tobacco-specific nitrosamine NNK in the A/J mouse revealed by proteomic analysis," Journal of Proteome Research, vol. 8, no. 8, pp. 4050-4061, 2009. 
[19] B. Ye, Y. X. Zhang, F. Yang et al., "Induction of lung lesions in Wistar rats by 4-(methylnitrosamino)-1-(3-pyridyl)-1butanone and its inhibition by aspirin and phenethyl isothiocyanate," BMC Cancer, vol. 7, article 90, 2007.

[20] H. M. Schuller, P. K. Tithof, M. Williams, and H. Plummer, "The tobacco-specific carcinogen 4-(methylnitrosamino)1-(3-pyridyl)-1- butanone is a $\beta$-adrenergic agonist and stimulates DNA synthesis in lung adenocarcinoma via $\beta$ adrenergic receptor-mediated release of arachidonic acid," Cancer Research, vol. 59, no. 18, pp. 4510-4515, 1999.

[21] C. L. K. Sabourin, Q. S. Wang, S. L. Ralston et al., "Expression of cell cycle proteins in 4-(methylnitrosamino)-1-(3- pyridyl)1-butanone-induced mouse lung tumors," Experimental Lung Research, vol. 24, no. 4, pp. 499-521, 1998.

[22] R. Ilona Linnoila, E. Szabo, F. Demayo, H. Witschi, C. Sabourin, and AL. Malkinson, "The role of CC10 in pulmonary carcinogenesis: from a marker to tumor suppression," Annals of the New York Academy of Sciences, vol. 923, pp. 249267, 2000.

[23] F. Kassie, L. B. Anderson, R. Scherber et al., "Indole3-carbinol inhibits 4-(methylnitrosamino)-1-(3-pyridyl)-1butanone plus benzo(a)pyrene-induced lung tumorigenesis in $\mathrm{A} / \mathrm{J}$ mice and modulates carcinogen-induced alterations in protein levels," Cancer Research, vol. 67, no. 13, pp. 6502-6511, 2007.

[24] L. P. Stabile, J. S. Lyker, S. R. Land, S. Dacic, B. A. Zamboni, and J. M. Siegfried, "Transgenic mice overexpressing hepatocyte growth factor in the airways show increased susceptibility to lung cancer," Carcinogenesis, vol. 27, no. 8, pp. 1547-1555, 2006.

[25] A. Ehrhardt, T. Bartels, R. Klocke, D. Paul, and R. Halter, "Increased susceptibility to the tobacco carcinogen 4(methylnitrosamino)-1-(3-pyridyl)-1-butanone in transgenic mice overexpressing c-myc and epidermal growth factor in alveolar type II cells," Journal of Cancer Research and Clinical Oncology, vol. 129, no. 2, pp. 71-75, 2003.

[26] R. A. Lubet, Z. Zhang, R. W. Wiseman, and M. You, "Use of p53 transgenic mice in the development of cancer models for multiple purposes," Experimental Lung Research, vol. 26, no. 8, pp. 581-593, 2000.

[27] M. D. Siwicky, J. J. Petrik, and R. A. Moorehead, "The function of IGF-IR in NNK-mediated lung tumorigenesis," Lung Cancer, vol. 71, no. 1, pp. 11-18, 2011.

[28] H. O. Abdel-Aziz, Y. Murai, I. Takasaki et al., "Targeted disruption of the galectin-3 gene results in decreased susceptibility to NNK-induced lung tumorigenesis: an oligonucleotide microarray study," Journal of Cancer Research and Clinical Oncology, vol. 134, no. 7, pp. 777-788, 2008.

[29] H. O. Abdel-Aziz, I. Takasaki, Y. Tabuchi et al., "High-density oligonucleotide microarrays and functional network analysis reveal extended lung carcinogenesis pathway maps and multiple interacting genes in NNK [4-(methylnitrosamino)1-(3-pyridyle)-1-butanone] induced CD1 mouse lung tumor," Journal of Cancer Research and Clinical Oncology, vol. 133, no. 2, pp. 107-115, 2007.

[30] L. Liu, X. Qin, and S. L. Gerson, "Reduced lung tumorigenesis in human methylguanine DNA-methyltransferase transgenic mice achieved by expression of transgene within the target cell," Carcinogenesis, vol. 20, no. 2, pp. 279-284, 1999.

[31] L. E. Sandercock, J. N. Hahn, L. Li et al., "Mgmt deficiency alters the in vivo mutational spectrum of tissues exposed to the tobacco carcinogen 4-(methylnitrosamino)-1-(3-pyridyl)1-butanone (NNK)," Carcinogenesis, vol. 29, no. 4, pp. 866$874,2008$.
[32] M. Igarashi, M. Watanabe, M. Yoshida et al., "Enhancement of lung carcinogenesis initiated with 4(N- hydroxymethylnitrosamino)-1-(3-pyridyl)-1-butanone by Ogg1 gene deficiency in female, but not male, mice," Journal of Toxicological Sciences, vol. 34, no. 2, pp. 163-174, 2009.

[33] M. C. Hollander, A. R. Balogh, J. Liwanag et al., "Strainspecific spontaneous and NNK-mediated tumorigenesis in Pten+/- mice," Neoplasia, vol. 10, no. 8, pp. 866-872, 2008.

[34] J. Fujimoto, H. Kadara, T. Men, C. van Pelt, D. Lotan, and R. Lotan, "Comparative functional genomics analysis of NNK tobacco-carcinogen induced lung adenocarcinoma development in Gprc5a-knockout mice," PLoS ONE, vol. 5, no. 7, Article ID e11847, 2010.

[35] Y. Y. Kim, L. von Weymarn, O. Larsson et al., "Eukaryotic initiation factor $4 \mathrm{E}$ binding protein family of proteins: sentinels at a translational control checkpoint in lung tumor defense," Cancer Research, vol. 69, no. 21, pp. 8455-8462, 2009.

[36] Y. Yang, Z. Zhang, A. B. Mukherjee, and R. I. Linnoila, "Increased susceptibility of mice lacking Clara cell $10-\mathrm{kDa}$ protein to lung tumorigenesis by 4 -(methylnitrosamino)1-(3-pyridyl)-1-butanone, a potent carcinogen in cigarette smoke," Journal of Biological Chemistry, vol. 279, no. 28, pp. 29336-29340, 2004.

[37] Y. Weng, C. Fang, R. J. Turesky, M. Behr, L. S. Kaminsky, and X. Ding, "Determination of the role of target tissue metabolism in lung carcinogenesis using conditional cytochrome P450 reductase-null mice," Cancer Research, vol. 67, no. 16, pp. 7825-7832, 2007.

[38] T. Kanehira, T. Tani, T. Takagi, Y. Nakano, E. F. Howard, and M. Tamura, "Angiotensin II type 2 receptor gene deficiency attenuates susceptibility to tobacco-specific nitrosamineinduced lung tumorigenesis: Involvement of transforming growth factor- $\beta$-dependent cell growth attenuation," Cancer Research, vol. 65, no. 17, pp. 7660-7665, 2005.

[39] B. Prokopczyk, J. G. Rosa, D. Desai et al., "Chemoprevention of lung tumorigenesis induced by a mixture of benzo(a)pyrene and 4-(methylnitrosamino)-1-(3-pyridyl)-1butanone by the organoselenium compound 1,4-phenylenebis(methylene)selenocyanate," Cancer Letters, vol. 161, no. 1, pp. 35-46, 2000.

[40] J. P. Richie Jr., W. Kleinman, D. H. Desai et al., "The organoselenium compound 1,4-phenylenebis(methylene)selenocyanate inhibits 4-(methylnitrosamino)-1-(3-pyridyl)-1butanone-induced tumorgenesis and enhances glutathionerelated antioxidant levels in A/J mouse lung," ChemicoBiological Interactions, vol. 161, no. 2, pp. 93-103, 2006.

[41] A. Das, D. Desai, B. Pittman, S. Amin, and K. El-Bayoumy, "Comparison of the chemopreventive efficacies of 1,4-phenylenebis(methylene)selenocyanate and selenium-enriched yeast on 4-(methylnitrosamino)-1-(3-pyridyl)-1-butanone induced lung tumorigenesis in $\mathrm{A} / \mathrm{J}$ mouse," Nutrition and Cancer, vol. 46, no. 2, pp. 179-185, 2003.

[42] L. Li, Y. Xie, W. M. El-Sayed, J. G. Szakacs, M. R. Franklin, and J. C. Roberts, "Chemopreventive activity of selenocysteine prodrugs against tobacco-derived nitrosamine (NNK) induced lung tumors in the A/J mouse," Journal of Biochemical and Molecular Toxicology, vol. 19, no. 6, pp. 396-405, 2005.

[43] J. H. Weisburger and F. L. Chung, "Mechanisms of chronic disease causation by nutritional factors and tobacco products and their prevention by tea polyphenols," Food and Chemical Toxicology, vol. 40, no. 8, pp. 1145-1154, 2002. 
[44] J. Liao, G. Y. Yang, E. S. Park et al., "Inhibition of lung carcinogenesis and effects on angiogenesis and apoptosis in A/J mice by oral administration of green tea," Nutrition and Cancer, vol. 48, no. 1, pp. 44-53, 2004.

[45] F. L. Chung, "The prevention of lung cancer induced by a tobacco-specific carcinogen in rodents by green and black tea," Proceedings of the Society for Experimental Biology and Medicine, vol. 220, no. 4, pp. 244-248, 1999.

[46] G. Lu, J. Liao, G. Yang, K. R. Reuhl, X. Hao, and C. S. Yang, "Inhibition of adenoma progression to adenocarcinoma in a 4-(methylnitrosamino)-1-(3-pyridyl)-1-butanone-induced lung tumorigenesis model in A/J mice by tea polyphenols and caffeine," Cancer Research, vol. 66, no. 23, pp. 11494-11501, 2006.

[47] T. Yano, S. Yajima, T. Nakamura, S. Horikawa, M. Kishimoto, and T. Ichikawa, "The inhibitory effect of vitamin $\mathrm{E}$ on 4-(methylnitrosamino)- 1-(3- pyridyl)-1-butanone-induced DNA injury and the fixation of the DNA injury in mouse lungs," Naunyn-Schmiedeberg's Archives of Pharmacology, vol. 358, no. 2, pp. 275-278, 1998.

[48] T. Yano, Y. Yano, S. Yajima et al., "The suppression of ornithine decarboxylase expression and cell proliferation at the promotion stage of lung tumorigenesis in mice by $\alpha$ tocopheryloxybutyric acid," Biochemical Pharmacology, vol. 61, no. 9, pp. 1177-1181, 2001.

[49] M. Kishimoto, Y. Yano, S. Yajima, S. Otani, T. Ichikawa, and T. Yano, "The inhibitory effect of vitamin E on 4(methylnitrosamino)-1-(3-pyridyl)-1-butanone-induced lung tumorigenesis in mice based on the regulation of polyamine metabolism," Cancer Letters, vol. 126, no. 2, pp. 173-178, 1998.

[50] G. Lu, H. Xiao, G. X. Li et al., "A $\gamma$-tocopherol-rich mixture of tocopherols inhibits chemically induced lung tumorigenesis in A/J mice and xenograft tumor growth," Carcinogenesis, vol. 31, no. 4, pp. 687-694, 2010.

[51] H. Mernitz, D. E. Smith, R. J. Wood, R. M. Russell, and X. D. Wang, "Inhibition of lung carcinogenesis by $1 \alpha, 25$ dihydroxyvitamin $\mathrm{D}$ and 9 -cis retinoic acid in the A/J mouse model: evidence of retinoid mitigation of vitamin D toxicity," International Journal of Cancer, vol. 120, no. 7, pp. 1402-1409, 2007.

[52] A. R. Dahl, I. M. Grossi, D. P. Houchens et al., "Inhaled isotretinoin (13-cis retinoic acid) is an effective lung cancer chemopreventive agent in A/J mice at low doses: a pilot study," Clinical Cancer Research, vol. 6, no. 8, pp. 3015-3024, 2000.

[53] H. Mernitz, D. E. Smith, A. X. Zhu, and X. D. Wang, "9cis-Retinoic acid inhibition of lung carcinogenesis in the A/J mouse model is accompanied by increased expression of RAR$\beta$ but no change in cyclooxygenase-2," Cancer Letters, vol. 244, no. 1, pp. 101-108, 2006.

[54] H. Mori, K. Matsunaga, Y. Tanakamaru et al., "Effects of protocatechuic acid, S-methylmethanethiosulfonate or 5-hydroxy-4-(2-phenyl-(E)ethenyl)-2(5H)-furanone(KYN-54) on 4(methylnitrosamino)-1-(3-pyridyl)-1-butanone-induced pulmonary carcinogenesis in mice," Cancer Letters, vol. 135, no. 2, pp. 123-127, 1999.

[55] S. Phutthaphadoong, Y. Yamada, A. Hirata et al., "Chemopreventive effects of fermented brown rice and rice bran against 4-(methylnitrosamino)-1-(3-pyridyl)-1-butanone-induced lung tumorigenesis in female A/J mice," Oncology Reports, vol. 21, no. 2, pp. 321-327, 2009.

[56] H. Y. Lee, S. H. Oh, J. K. Woo et al., "Chemopreventive effects of deguelin, a novel Akt inhibitor, on tobacco-induced lung tumorigenesis," Journal of the National Cancer Institute, vol. 97, no. 22, pp. 1695-1699, 2005.

[57] H. C. Chang, Y. C. Huang, and W. C. Hung, "Antiproliferative and chemopreventive effects of adlay seed on lung cancer in vitro and in vivo," Journal of Agricultural and Food Chemistry, vol. 51, no. 12, pp. 3656-3660, 2003.

[58] J. L. Kyung, W. S. Dong, C. C. Young, and G. J. Hye, "Chemopreventive effect of saponins derived from roots of Platycodon grandiflorum on 4-(methylnitrosamino)-1(3-pyridyl)-1-butanone-induced lung tumorigenesis in $\mathrm{A} / \mathrm{J}$ mice," Archives of Pharmacal Research, vol. 29, no. 8, pp. 651656, 2006.

[59] J. H. Kim, H. J. Lee, G. S. Kim et al., "Inhibitory effects of 7hydroxy-3-methoxy-cadalene on 4- (methylinitrosamino)-1(3-pyridyl)-1-butanone (NNK)-induced lung tumorigenesis in A/J mice," Cancer Letters, vol. 213, no. 2, pp. 139-145, 2004.

[60] T. E. Johnson, F. Kassie, M. G. O’Sullivan et al., "Chemopreventive effect of kava on 4-(methylnitrosamino)1-(3-pyridyl)-1-butanone plus benzo[a]pyrene-induced lung tumorigenesis in A/J mice," Cancer Prevention Research (Philadelphia, Pa.), vol. 1, no. 6, pp. 430-438, 2008.

[61] C. C. Conaway, C. X. Wang, B. Pittman et al., "Phenethyl isothiocyanate and sulforaphane and their $\mathrm{N}$-acetylcysteine conjugates inhibit malignant progression of lung adenomas induced by tobacco carcinogens in A/J mice," Cancer Research, vol. 65 , no. 18, pp. 8548-8557, 2005.

[62] F. L. Chung, "Chemoprevention of lung cancer by isothiocyanates and their conjugates in A/J mouse," Experimental Lung Research, vol. 27, no. 3, pp. 319-330, 2001.

[63] H. Kohno, M. Taima, T. Sumida, Y. Azuma, H. Ogawa, and T. Tanaka, "Inhibitory effect of mandarin juice rich in $\beta$ cryptoxanthin and hesperidin on 4-(methylnitrosamino)-1(3-pyridyl)-1-butanone-induced pulmonary tumorigenesis in mice," Cancer Letters, vol. 174, no. 2, pp. 141-150, 2001.

[64] H. A. N. Al-Wadei and H. M. Schuller, " $\beta$-carotene promotes the development of NNK-induced small airway-derived lung adenocarcinoma," European Journal of Cancer, vol. 45, no. 7, pp. 1257-1264, 2009.

[65] S. A. Matzinger, K. A. Crist, G. D. Stoner et al., "Kras mutations in lung tumors from A/J and A/JxTSG-p53 F mice treated with 4-(methylnitrosamino)-1-(3-pyridyl)-1butanone and phenethyl isothiocyanate," Carcinogenesis, vol. 16, no. 10, pp. 2487-2492, 1995.

[66] M. Yokohira, H. Takeuchi, K. Saoo et al., "Establishment of a bioassay model for lung cancer chemoprevention initiated with 4-(methylnitrosamino)-1-(3-pyridyl)-1-butanone (NNK) in female A/J mice," Experimental and Toxicologic Pathology, vol. 60, no. 6, pp. 469-473, 2008.

[67] H. Takeuchi, K. Saoo, M. Yokohira et al., "Pretreatment with 8-methoxypsoralen, a potent human CYP2A6 inhibitor, strongly inhibits lung tumorigenesis induced by 4 (methylnitrosamino)-1-(3-Pyridyl)-1-butanone in female A/J mice," Cancer Research, vol. 63, no. 22, pp. 7581-7583, 2003.

[68] T. Kuno, M. Yokohira, Y. Matsuda et al., "Lack of modifying potential of 8-methoxypsoralen in the promotion or progression stages of lung carcinogenesis in A/J female mice," Oncology Reports, vol. 20, no. 4, pp. 767-772, 2008.

[69] T. Kamataki, M. Fujieda, K. Kiyotani, S. Iwano, and H. Kunitoh, "Genetic polymorphism of CYP2A6 as one of the potential determinants of tobacco-related cancer risk," Biochemical and Biophysical Research Communications, vol. 338, no. 1, pp. 306-310, 2005. 
[70] H. M. Schuller, G. Kabalka, G. Smith, A. Mereddy, M. Akula, and M. Cekanova, "Detection of overexpressed COX2 in precancerous lesions of hamster pancreas and lungs by molecular imaging: implications for early diagnosis and prevention," ChemMedChem, vol. 1, no. 6, pp. 603-610, 2006.

[71] R. Yao, N. Rioux, A. Castonguay, and M. You, "Inhibition of COX-2 and induction of apoptosis: Two determinants of nonsteroidal anti-inflammatory drugs' chemopreventive efficacies in mouse lung tumorigenesis," Experimental Lung Research, vol. 26, no. 8, pp. 731-742, 2000.

[72] L. E. Lantry, Z. Zhang, K. A. Crist et al., "Chemopreventive efficacy of promising farnesyltransferase inhibitors," Experimental Lung Research, vol. 26, no. 8, pp. 773-790, 2000.

[73] H. Mernitz, F. Lian, D. E. Smith, S. N. Meydani, and X. D. Wang, "Fish oil supplementation inhibits NNK-induced lung carcinogenesis in the A/J mouse," Nutrition and Cancer, vol. 61, no. 5, pp. 663-669, 2009.

[74] T. Yamaki, T. Yano, H. Satoh et al., "High oleic acid oil suppresses lung tumorigenesis in mice through the modulation of extracellular signal-regulated kinase cascade," Lipids, vol. 37, no. 8, pp. 783-788, 2002.

[75] H. Witschi, I. Espiritu, M. Ly, and D. Uyeminami, “The effects of dietary myoinositol on lung tumor development in tobacco smoke-exposed mice," Inhalation Toxicology, vol. 16, no. 4, pp. 195-201, 2004.

[76] D. Kishino, K. Kiura, N. Takigawa et al., "Effect of gefitinib on N-nitrosamine-4-(methylnitrosamino)-1-(3-pyridyl)-1butanone induced lung tumorigenesis in A/J mice," Lung Cancer, vol. 65, no. 3, pp. 284-289, 2009.

[77] C. A. Granville, N. Warfel, J. Tsurutani et al., "Identification of a highly effective rapamycin schedule that markedly reduces the size, multiplicity, and phenotypic progression of tobacco carcinogen-induced murine lung tumors," Clinical Cancer Research, vol. 13, no. 7, pp. 2281-2289, 2007.

[78] M. A. Pereira, P. M. Kramer, R. Nines et al., "Prevention of mouse lung tumors by targretin," International Journal of Cancer, vol. 118, no. 9, pp. 2359-2362, 2006.

[79] D. Desai, A. Das, L. Cohen, K. El-Bayoumy, and S. Amin, "Chemopreventive efficacy of suberoylanilide hydroxamic acid (SAHA) against 4-(methylnitrosamino)-1-(3-pyridyl)-1butanone (NNK)-induced lung tumorigenesis in female A/J mice," Anticancer Research, vol. 23, no. 1A, pp. 499-503, 2003. 


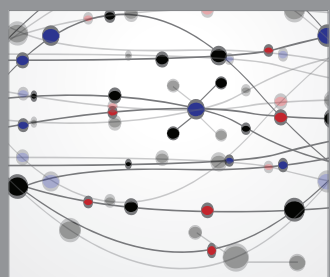

The Scientific World Journal
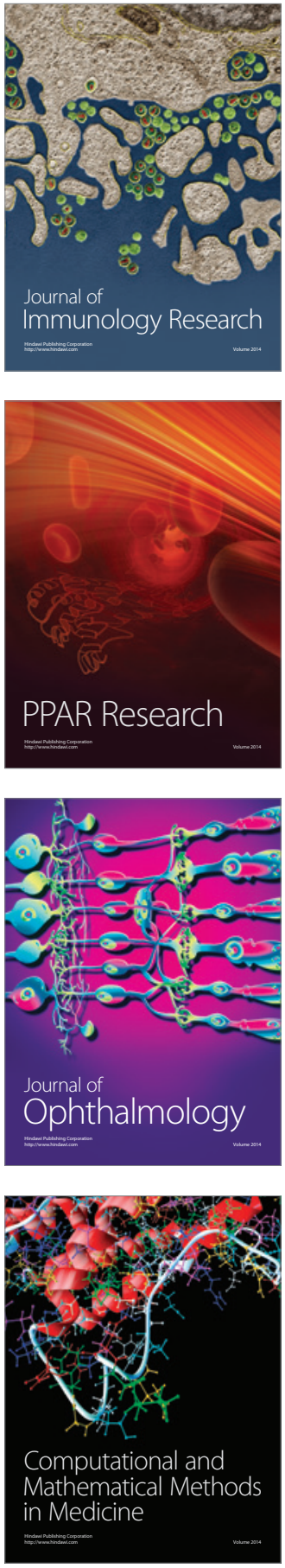

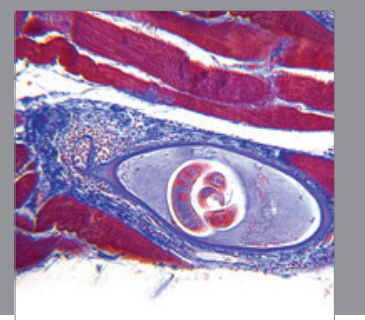

Gastroenterology

Research and Practice
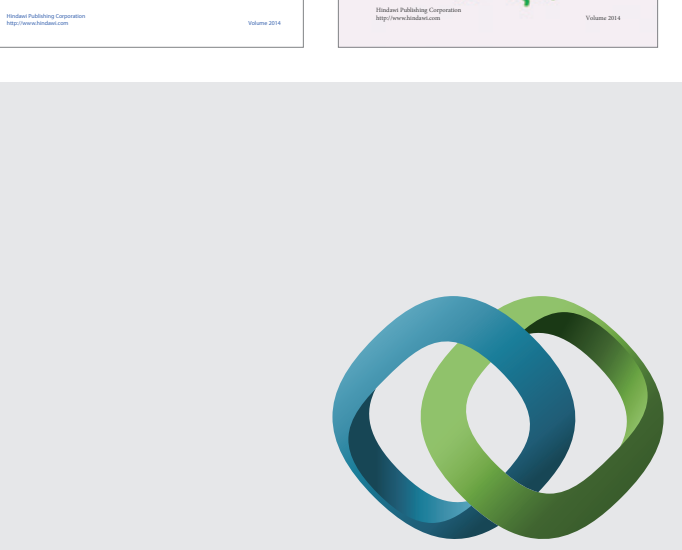

\section{Hindawi}

Submit your manuscripts at

http://www.hindawi.com
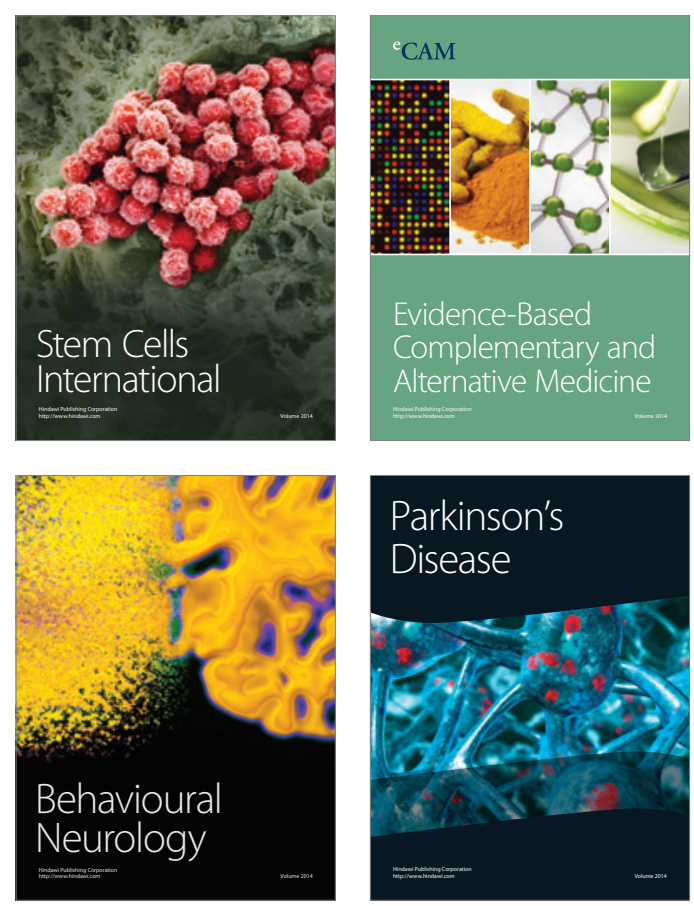

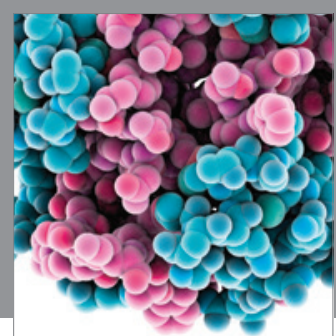

Journal of
Diabetes Research

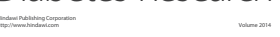

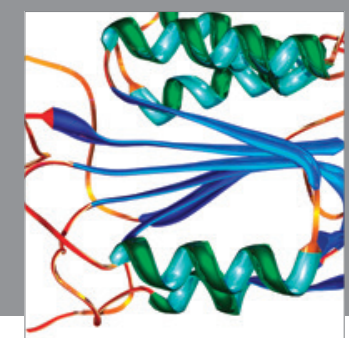

Disease Markers
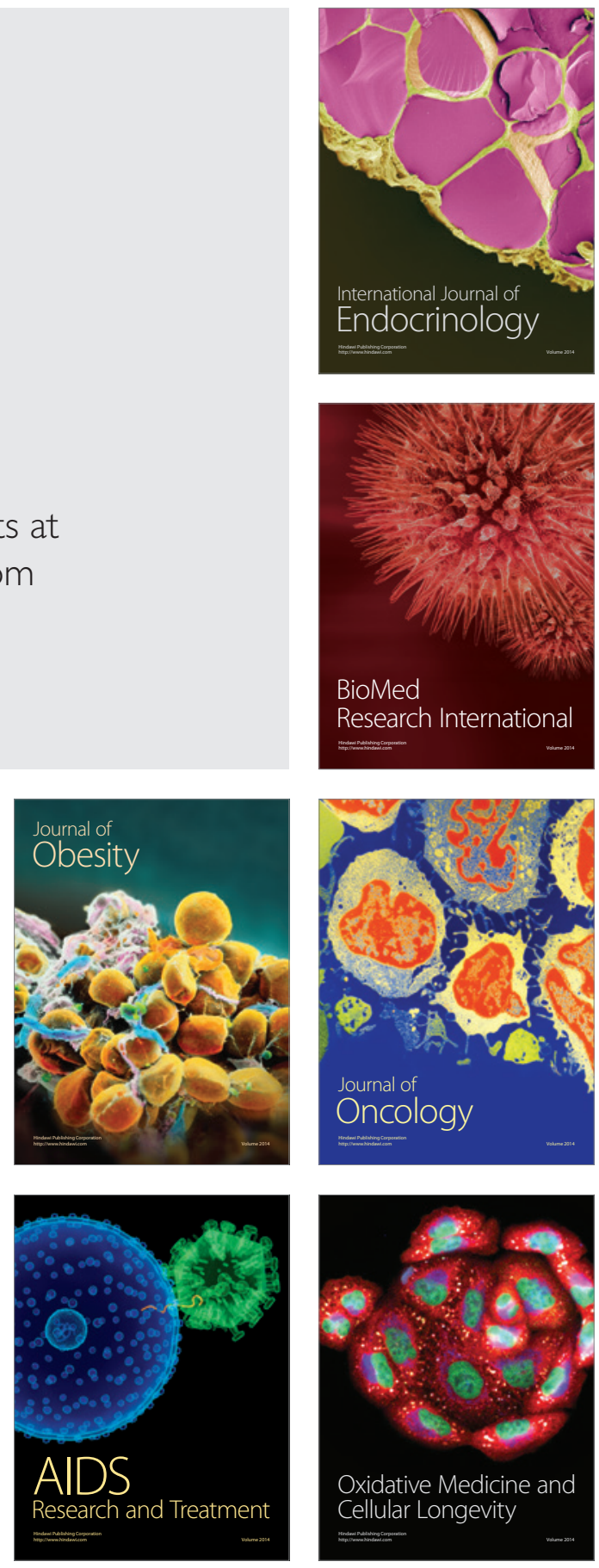